Casos Clínicos

Arch. Esp. Urol., 61, 7 (837-840), 2008

\title{
ENFERMEDAD DE MONDOR VERSUS LINFANGITIS ESCLEROSANTE DE PENE
}

Enrique Ramos Barseló, José Antonio Portillo Martín, Miguel Correas Gomez, José Luis Gutierrez Baños, Carmen Aguilera Tubet, Roberto Ballestero Diego, Sergio Zubillaga Guerrero, José Antonio Campos Sañudo, Emma Hidalgo Zabala, Pedro Lastra GarcíaBarón' y Gerardo Lopez Rasines'.

Servicio de Urología. Servicio de Radiodiagnóstico'. Hospital Universitario Marqués de Valdecilla. Facultad de Medicina. Universidad de Cantabria. Santander. España.

Resumen.- OBJETIVO: Resaltar la diferencia entre Enfermedad de Mondor y Linfangitis Esclerosante del Pene mediante el análisis de tres casos clínicos y la revisión de la literatura existente.

MÉTODOS: Describimos dos casos clínicos compatibles con el diagnóstico de enfermedad de Mondor y otro compatible con Linfangitis.

RESULTADO: La buena y muchas veces espontánea resolución de estas dos patologías dificulta el diagnóstico y seguimiento de estos pacientes.

Enrique Ramos Barseló

Servicio de Urología

Hospital Universitario Marqués de Valdecilla

Avda. Valdecilla sn.

39008 Santander. Cantabria. (España).

enriqueramosbarselo@hotmail.com

Trabajo recibido: 29 de noviembre 2007. 
CONCLUSIONES: La abstinencia sexual es una de las medidas más efectiva para la pronta resolución de ambos procesos. Para el diagnóstico diferencial entre ambas entidades es de gran utilidad el eco-doppler peneano. El tratamiento farmacológico principal se basa en antiinflamatorios.

Palabras clave: Enfermedad de Mondor. Linfangitis esclerosante peneana.

Summary.- OBJECTIVE: Two cases of Mondor's disea-se and one case of non-venereal sclerosing lymphangitis of the penis are reviewed. We analyze the differences bet ween both processes based on the existing literature about these pathologies.

METHOD: Two clinical cases of Mondor's disease and other one with non-venereal sclerosing lymphangitis are reported.

RESULTS: Due to the spontaneous and good outcome of both pathologies, the diagnosis and follow-up are difficult in both processes.

CONCLUSIONS: Doppler ultrasound has a great importance for the differential diagnosis between both processes. The treatment is based on steroidal anti-inflammatories as well as sexual abstinence.

Keywords: Mondor's Disease. Sclerosing Lymphangitis of penis.

\section{INTRODUCCIÓN}

La trombosis de las venas superficiales de la pared torácica fue descrita por primera vez por Mondor en 1939. En 1955 Braun-Falcó aplicó este término a la trombosis de la vena dorsal del pene en el contexto de una flebitis generalizada (1). Fueron Helm y Hodge quienes en 1958 describieron en forma individualizada la afectación peneana. Cinco años más tarde Harrow y Sloane publicaron el segundo artículo sobre dicha entidad.

Es importante diferenciar la Enfermedad trombosis de la vena dorsal superficial del pene, del síndrome de Mondor, síndrome toxémico-hemolítico que sigue a un aborto séptico provocado o espontáneo. Suele aparecer dentro de las 24-48 horas posteriores al aborto, es extremadamente grave, presenta una tasa de mortalidad del $60 \%$ y es provocado por gérmenes anaeróbicos esporulados (Clostridium Perfringens) en el $80 \%$ de los casos que como resultado de la endotoxemia ocasiona CID, acidosis metabólica importante, insuficiencia renal aguda y shock.
La Linfangitis Esclerosante del pene, linfangiectasia esclerosante no venérea o linfangiectasia transitoria benigna del pene, fue descrita por primera vez por Hoffman en 1923, quien más tarde la denominó linfangitis plástica no venérea del surco coronal ya que el propio Hoffman al principio lo consideró una consecuencia de la sífilis primaria por linfangitis blenorrágica (pseudochancro gonorreico), pero quince años más tarde evidenció que no existía correlación entre esta lesión y la enfermedad venérea por lo que le cambió la definición (2). Von Berde la describió como linfangitis circular indurada. En 1962 Nickel y Plumb revisaron la literatura y aportaron tres casos más haciendo una descripción más amplia de la patología.

\section{CASOS CLÍNICOS}

\section{CASO 1}

Varón de 30 años sin antecedentes de interés que acude a Urgencias por presentar desde hace una semana una induración dolorosa en el pene que se incrementa con la erección. El paciente refería aumento de la actividad sexual en días previos. A la exploración presentaba una zona eritematosa y dolorosa en forma concéntrica un centímetro por debajo del surco balanoprepucial en zona dorsal (Figura 1). No se palpaban adenopatías inguinales. Afebril.

Se realizó eco-doppler peneano que evidenció una reacción inflamatoria a nivel de los linfáticos con permeabilidad de la vena dorsal del pene (Figura 2). Estudio de coagulación normal.

Juicio diagnóstico: Linfangitis Esclerosante. El paciente recibió tratamiento con antiinflamatorios, estando a las tres semanas asintomático.

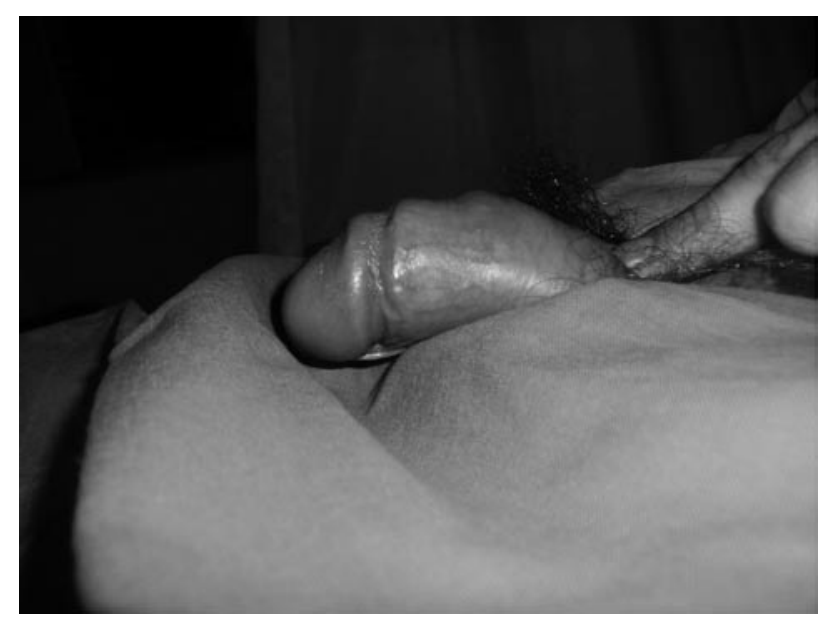

FIGURA 1. Reacción eritematosa paralela al surco balano prepucial encontrada frecuentemente en LEP. 


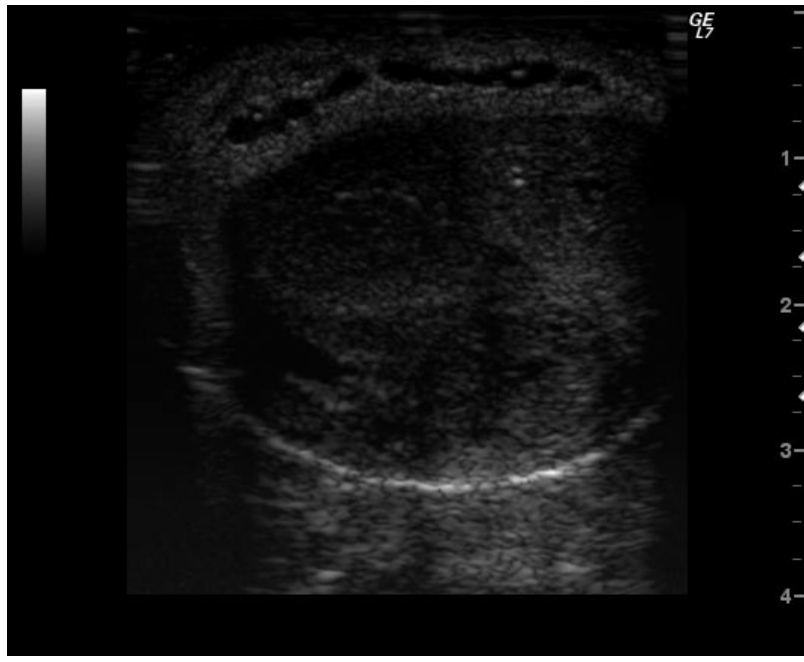

FIGURA 2. Imagen compatible con LEP. No se evidencia trombosis venosa.

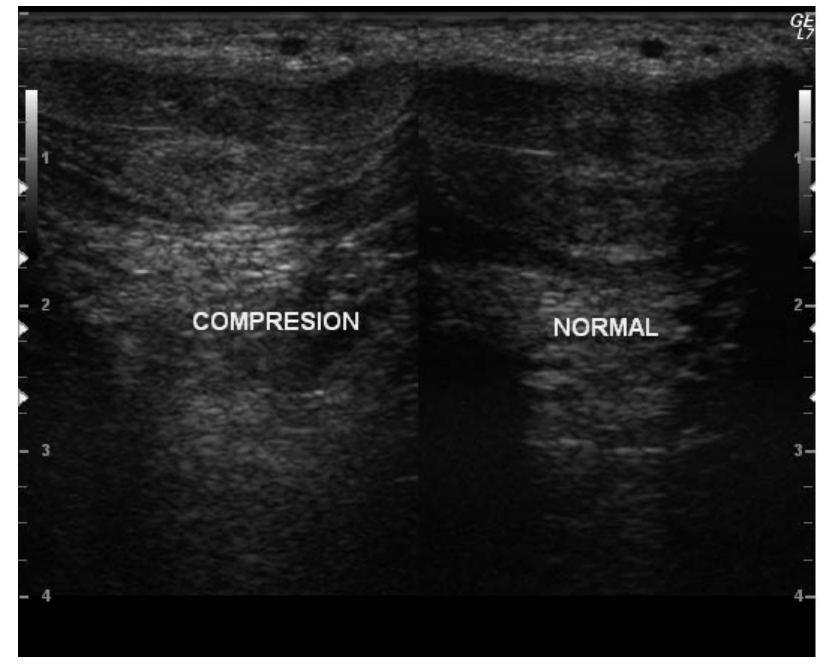

FIGURA 3. Trombosis de la vena dorsal superficial del pene. No se consigue colapsar a la presión del transductor.

\section{CASO 2}

Varón de 33 años fumador de un paquete de cigarrillos al día sin otros antecedentes de interés que acude a Urgencias por presentar desde hacía diez días induración peneana a nivel de la base. A la exploración se evidenció cordón longitudinal indurado en la base del pene de localización dorsal, móvil y doloroso. No se palparon adenopatías inguinales. Afebril. Se realizó eco-doppler peneano en el que se objetivó una induración correspondiente a trombosis segmentaria de la vena dorsal superficial del pene. Estudio de coagulación normal.

Juicio Diagnóstico: Enfermedad de Mondor. El paciente recibió tratamiento antiinflamatorio. No acudió a la revisión.

\section{CASO 3}

Varón de 37 años al que un mes antes se le había realizado una hemorragia bilateral. Resto de antecedentes sin interés. Acude a Urgencias por presentar desde hace 10 días un bulto en el pene, doloroso con la erección. A la exploración presentaba un cordón longitudinal en el dorso del pene de unos tres centímetros, móvil y doloroso. Afebril. No adenopatías inguinales. Se realizó eco-doppler peneano en el que se evidenció una clara trombosis de la vena dorsal superficial del pene y que no se colapsaba a la presión del transductor (Figura 3). Estudio de coagulación normal.

Juicio Diagnóstico: Enfermedad de Mondor. El paciente recibió tratamiento con heparina de bajo peso molecular y antiinflamatorios.

\section{DISCUSIÓN}

La Enfermedad de Mondor (EM) se presenta en hombres entre 21-70 años (3). Revisiones posteriores delimitan más la edad encontrando en series más grandes que la patología se presenta principalmente por debajo de los 45 años. La edad de presentación de la Linfangitis Esclerosante del Pene (LEP) es entre 20-40 años.

Los factores predisponentes de la EM incluyen principalmente la prolongada actividad sexual, traumatismos incluyendo los microtraumatismos de repetición, abstinencia sexual prolongada, tumores pélvicos, infecciones, hipospadias con o sin relación con su corrección quirúrgica, sobredistensión vesical, cirugía de hernia inguinal, trombosis venosa profunda, elementos constrictores utilizados en determinadas prácticas sexuales, drogas endovenosas (4). La LEP tiene una etiología controvertida entre la que se encuentran factores traumáticos por relaciones sexuales enérgicas, masturbación, (infecciones por microorganismos como Clamydia Trachomatis, Mycobacterium Tuberculosis, Herpes Simple...). También señalar variaciones anatómicas de drenaje linfático que predisponen a la lesión.

De aquí la importancia de una buena anamnesis y exploración física a estos pacientes para no pasar por alto patologías que puedan ser de relevancia.

Ambas entidades tienen una prevalencia muy baja difícilmente cuantificable ya que muchos de estos pacientes no llegan a acudir al médico. Su pronóstico favorable y de resolución espontánea, restitución ad integrum en 8-10 semanas en el caso de EM, hace que estos pacientes no lleguen muy a menudo a la consulta del urólogo. 
La evolución de la EM incluye una fase aguda, subaguda, crónica y recanalización. La fase aguda suele ocurrir 24-48 horas tras una actividad sexual prolongada. A la exploración la EM se presenta como un cordón indurado y doloroso de localización dorsal. Sin embargo, la LEP aparece como unos nódulos móviles de consistencia cartilaginosa paralelos al surco balano-prepucial que coalescen y pueden llegar a formar un cordón.

Desde el punto de vista ecográfico en la EM se observa un incremento del calibre de la vena dorsal del pene, falta de compresibilidad y lesión endoluminal hipoecoica (5). Las paredes de la vena presentan cambios inflamatorios. El doppler muestra reducción o ausencia del flujo venoso. En cambio en la LEP la vena dorsal superficial está permeable en todo su trayecto y los cambios inflamatorios se visualizan a nivel linfático. Otros autores, en cambio, no consideran el eco-doppler útil para establecer el diagnóstico (6).

El diagnóstico diferencial debe establecerse entre estas dos patologías y con la Enfermedad de Peyronie, la cual es un cuadro clínico en el que se forman placas fibróticas en la albugínea del pene que en sus formas más graves incurva el pene hasta imposibilitar la penetración. También se debe diferenciar de la angeitis subaguda que puede presentarse en el contexto de una PAN o por drogas (7). El diagnóstico diferencial entre EM y LEP es bastante controvertido. Muchos autores incluso desacreditan una $u$ otra entidad (8) y otros refieren ser la misma e incluso lo llaman Linfangitis Esclerosante de Mondor (9). La forma más objetiva de llegar a un diagnóstico diferencial adecuado sería con técnicas de inmunohistoquímica para lo cual haría falta una biopsia. Dicha biopsia y su estudio se realizan de forma infrecuente, resultando una técnica invasiva que no está indicada de rutina. Marsch y Stüttgen biopsiaron y analizaron al microscopio electrónico muestras de pacientes con clínica compatible con LEP encontrando una linfangiofibrosis trombótica oclusiva en todas las muestras (10).

\section{CONCLUSIONES}

El tratamiento de la EM es principalmente sintomático con antiinflamatorios. La utilización de drogas anticoagulantes $y / o$ antiagregantes no ha demostrado modificar el curso de la enfermedad. Si no está asociado a infección, la antibioterapia tampoco es de utilidad (10). Dependiendo de la fase varía el tratamiento: en la fase aguda antiinflamatorios y anticoagulantes. En la fase subaguda y crónica se pueden utilizar antiinflamatorios y cremas locales con heparina. En casos persistentes puede estar indicada la trombectomía o resección de la vena dorsal del pene, lo cual suele ser excepcional (11). En la LEP se recomienda actitud expectante, siendo lo más importante la abstinencia sexual hasta que se resuelva el proceso de forma espontánea. Sólo en muy determinados casos de persistencia de la lesión o ser muy recidivante está indicado la extirpación quirúrgica del cordón linfático.

\section{BIBLIOGRAFIA y LECTURAS RECOMENDADAS (*lectura de interés $y^{* *}$ lectura fundamental)}

**1. BRAUN-FALCO, O.: "Zur klinik, Histologie und Pathogenese der strangförmigen oberflächlichen Phlebitiden". Derm. Wochenschr., 132: 705, 1955.

*2. HOFFMANN, E.: "Uber nich venerische plastiche Lymphangitis in sulcus coronatius penis mit umschriebenem". Dermat. Ztschr., 78: 24, 1938.

3. RODRÍGUEZ FABA, O.; PARRA MUNTANER, L.; GOMEZ CISNEROS, S.C. y cols.: "Trombosis de la vena dorsal del pene (Flebitis de Mondor). Aportación de un nuevo caso". Actas Urol. Esp., 30: 80, 2006.

*4. OZKARA, H.; AKKUS, E.; ALICI, B. y cols.: "Superficial dorsal penile vein trombosis (penile Mondor's disease)". Int. Urol. Nephrol., 28: 387, 1996.

5. ROMBOLÁ, S.E.; BERESÑAC, A.: "Tromboflebitis superficial del pene. Enfermedad de Mondor". Rev. Argent. Radiol. 69: 169, 2005.

**6. DICUIO, M.; POMARA, G.; ALES, V. y cols.: "Doppler ultrasonography in a young patient with penile Mondor's disease". Archivio Italiano di Urologia e Andrologia, 77: 1, 2005.

7. KUMAR, B.; NARANG, T.; RADOTRA, D. y cols.: "Mondor's Disease of penis: a forgotten disease". Sex. Transm. Infect., 81: 480, 2005.

*8. BALL, T.P.; PICKETT, M.J.: "Traumatic lymphangitis of penis". Urology, 6: 5, 1975.

*9. PIQUERO-CASALS, J.; PIQUERO-MARTÍN, J.; HERNÁNDEZ-PEREZ, R.: "Linfangitis esclerosante del pene. A propósito de dos casos". Dermatología Venezolana, 43: 3, 2005.

**10. MARSCH, W.; STÜTTGEN, G.: "Sclerosing lymphangitis of the penis: A lymphangiofibrosis thrombotica occlusive”. British Journal of Dermatology, 104, 687, 1981.

**11. KRAUS, S.; LÜDECKE, G.; WEIDNER, W.: "Mondor's Disease of the penis". Urol. Int., 64: 99, 2000 . 